This item was submitted to Loughborough's Research Repository by the author.

Items in Figshare are protected by copyright, with all rights reserved, unless otherwise indicated.

\title{
An ecological momentary assessment of lapse occurrences in dieters
}

PLEASE CITE THE PUBLISHED VERSION

http://dx.doi.org/10.1007/s12160-014-9594-y

\section{PUBLISHER}

Springer (@ The Society of Behavioral Medicine)

\section{VERSION}

AM (Accepted Manuscript)

\section{LICENCE}

CC BY-NC-ND 4.0

\section{REPOSITORY RECORD}

McKee, Heather C., Nikos Ntoumanis, and lan M. Taylor. 2019. "An Ecological Momentary Assessment of Lapse Occurrences in Dieters”. figshare. https://hdl.handle.net/2134/15379. 
This item was submitted to Loughborough's Institutional Repository (https://dspace.lboro.ac.uk/) by the author and is made available under the following Creative Commons Licence conditions.

\section{creative
commons}

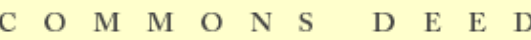

Attribution-NonCommercial-NoDerivs 2.5

You are free:

- to copy, distribute, display, and perform the work

Under the following conditions:

Attribution. You must attribute the work in the manner specified b the author or licensor.

Noncommercial. You may not use this work for commercial purposes.

No Derivative Works. You may not alter, transform, or build upon this work.

- For any reuse or distribution, you must make clear to others the license terms of this work.

- Any of these conditions can be waived if you get permission from the copyright holder.

Your fair use and other rights are in no way affected by the above.

This is a human-readable summary of the Leqal Code (the full license).

\section{Disclaimer 만}

For the full text of this licence, please go to: http://creativecommons.org/licenses/by-nc-nd/2.5/ 
Running head: LAPSE OCCURRENCES IN DIETERS

An Ecological Momentary Assessment of Lapse Occurrence in Dieters 


\begin{abstract}
Purpose: To investigate the factors related to dietary lapse occurrence in a community sample of dieters. Methods: An ecological momentary assessment (EMA) methodology, via mobile phone-based diaries was employed to record dietary lapse occurrences in a group of dieters $\left(N=80 ; M_{\text {age }}=41.21 \pm 15.60\right.$ years; $\left.M_{\mathrm{BMI}}=30.78 \pm 7.26\right)$ over seven days. Results: Analyses indicated that lapses were positively associated with the strength of dietary temptation, presence of others, coping responses, and the environment (exposure to food cues) in which the dieters were in; lapses were more likely to occur in the evening and were negatively associated with the use of coping mechanisms. Additionally, lapse occurrence was found to mediate the relationships among the above predictors of lapse and the self-efficacy to resist future dietary temptations. Conclusions: Results provide an insight into the occurrence of lapses in dieters and have implications for interventions focusing on weight loss maintenance and relapse prevention.
\end{abstract}

Keywords: Lapse, dietary temptation, self-control, weight maintenance, ecological momentary assessment. 
An Ecological Momentary Assessment of Lapse Occurrence in Dieters

Regardless of the increased knowledge, attention, and interest surrounding obesity, maintaining weight loss remains an unattainable goal for many individuals [1]. As knowing the means by which to lose weight appears to be insufficient for successful long-term weight maintenance, researchers have called for a focus on the psychological factors that lead to successful weight maintenance [2]. One such factor is the ability to resist short-term temptations in order to pursue long-term goals. This is known as willpower and is increasingly acknowledged as a key element influencing successful weight management [3]. Willpower is a term used interchangeably with self-control to describe the effortful regulation of the self by the self [4]. 'Lack of willpower' has been ranked by British dieticians as more important to the development of obesity than genetic factors [5]. Determining why individuals' willpower or self-control fails is thus crucial to help prevent dietary relapse and preserve weight maintenance success. The current study brings together a number of intrapersonal, situational and psychological variables that have been proposed in the literature as factors linked to the ability to self-control. All these factors have the common element of exerting their influence by strengthening or weakening self-control to resist temptations, including dietary ones. Our work is theory-driven but it is not based on one theoretical framework; this would have been too limiting given the broad range of factors that affect the ability to resist temptation.

The role of self-control in dealing with temptations, including dietary temptations (i.e. a sudden urge to overeat or eat a forbidden food, in which one felt they had come close to the brink of breaking their diet [6]), has mainly been examined in laboratory conditions. These lab-based studies have found that self-control resources are limited and that repeated exertions of self-control can deplete one's ability to resist future temptation. However, these studies mainly examined self-control using tasks such as hand grip strength or numerical 
accuracy and thus are limited in their generalizability [7]. Indeed, there is minimal research concerning the operation of dietary temptations in everyday life [8]. Additionally the majority of field-based research to date has been overly reliant on retrospective data. Such data can underestimate the strength or frequency of dietary lapse (i.e., an incident where one felt that they had broken their diet (e.g., overate, ate an unhealthy food, etc. [6]) as such characteristics are often subject to recall bias and inaccuracies [9]. The small number of studies investigating how dietary temptations operate outside laboratory conditions have sought to overcome the problems associated with retrospective recall by employing "real time” methods, such as ecological momentary assessment (EMA). EMA using electronic diaries or phones in particular (as opposed to employing paper and pencil diaries) is a data collection technique which allows for multiple, repeated, immediate reports of people and their activities in their natural everyday environment [9]. EMA methods are particularly suited to study daily dietary temptations as they are able to capture the immediate and often fleeting nature of a self-control dilemma (e.g., a dieter trying to decide whether or not to forgo the dessert course in a restaurant).

EMA studies $[6,10]$ have highlighted the importance of examining a number of daily intrapersonal (e.g. perceived strength of temptations), situational (e.g., presence of others), and psychological (e.g. coping responses) factors, and to a lesser extent, general dispositions (e.g. self-control) related to dietary temptation and lapse. Carels et al. [10] examined dietary lapse instances in a group of women $(N=37)$ in the final week of a weight-loss intervention. They found that coping responses and mood determined whether an individual gave in to a lapse or not. However, dietary lapses were limited in number $(M=2.7, S D=1.9)$, which the authors felt was a potential drawback of using paper-and-pencil diaries. Using a signal-event approach, Hofmann et al. [8] tested a four-step conceptual model which integrated desire/temptation strength, conflict with other goals, resistance to the temptation, and 
enactment (giving in to temptation). This model suggests that whether a person will give in to temptation depends on a) how strong the temptation is, b) the level of conflict experienced with other goals, which is dependent on the strength of the temptation, and c) the resistance (degree of use of self-control) to the temptation, which is dependent on the level of conflict experienced. Using a sample of 205 university students, Hofmann et al. [8] instructed these participants to record on electronic personal data assistants (PDAs) a multitude of "everyday desires/temptations” they experienced over a period of a week. Each time they received a random beeper message, participants recorded on the PDAs whether or not they were experiencing a desire, its strength, whether and how it conflicted with other goals, and whether they gave in to the desire. Results generally confirmed the hypothesis of the fourstep model in that as desire strength increased, an individual was less able to resist giving in to that desire. Additionally, results indicated that desire strength, conflict with one's goals, resistance and lapse were differentially predicted by situational (e.g. the presence of others) and dispositional variables (e.g. self-control). The aim of the Hofmann et al. [8] study was to document "everyday desires/temptations" and thus it had a broad focus on a number of different types of temptation (eating, drinking, socializing, and sleeping). Therefore, it is not possible to determine from this study the specific variables that relate to dietary temptations alone. The current study aims to build on the above findings by using electronic EMA methods to assess the daily factors associated with lapse occurrence in a community sample of dieters.

As stated earlier, and in alignment with other EMA studies [e.g., 8, 10], we did not rely exclusively on one conceptual framework. The self-control needed to successfully pursue long-term goals may be influenced by a multitude of daily fluctuating factors (intrapersonal, situational and psychological; presented in italics below) which can determine whether an individual will eventually lapse or not. By examining these different perspectives we hope to 
provide a coherent, socio-ecological framework. At the core of this framework are intrapersonal variables which influence one's ability to resist temptation; these variables have been taken from Baumeister's self-control model [11] and related work. Specifically, repeated exertions of self-control fatigue or deplete one’s limited self-control resource and hence one's ability to continually resist dietary temptations [11]. This depletion may lead to a greater likelihood of lapse occurrence later in the day. In addition, levels of stress have been linked with increased food consumption, possibly due to poor coping responses [12]. Coping with stressors requires an individual to override or stop thoughts, urges and emotions and thus uses up self-control strength [13]. Moreover, running low on energy (i.e. being in a state of hunger) may have an impact on one's ability to resist dietary temptation [6], due to depletion effects caused by drops in blood glucose levels [13]. These three intrapersonal factors (depletion, stress, and hunger) are likely to shape individuals’ perceived dietary temptation strength, which has been shown to predict lapse occurrence [8].

Building on these intrapersonal factors, it is also important to consider one's psychological resources available during tempting episodes. For example, coping responses can distinguish between successfully dealing with dietary temptation and lapsing $[10,15]$. In a tempting situation individuals may try to reduce their valuation and strength of temptation (e.g., high calorie snack) whilst at the same time boost their valuation of their focal goal (e.g., diet) in order to protect the latter [16]. Fishbach et al.’s self-regulation research on goals and temptations [16] also demonstrated that those who think of their long-term weight loss goal, in addition to the importance of that goal, when faced with dietary temptation are less likely to lapse. However, to date such coping responses have only been measured in relation to a single isolated incidence of dietary temptation.

Besides personal/psychological factors, our framework also considers external (situational) factors that could be instrumental in shaping dietary temptation episodes and 
lapses. For example, Wansink's work on mindless eating has indicated that the presence of others in social situations where food is available can contribute to increased consumption by increasing temptation strength [14]. Additionally, a person's environment (i.e. their exposure to food cues) has been associated with overeating [14]. Those with dieting goals may make efforts to control their environment by removing tempting foods and avoiding situations where they feel vulnerable to dietary temptation, however, unexpected exposure to dietary temptations may offset their self-control capabilities [11].

Drawing from the aforementioned gaps in the existent literature, the current study aims to synthesize some of the diverse intrapersonal characteristics, psychological resources, and situational daily fluctuating factors that have been previously associated with weight loss success and failure, and investigate whether these variables can also predict the strength of dietary temptations and lapse occurrences. By integrating these three types of predictors, we attempted to provide a socio-ecological explanation of dietary temptation and lapse occurrence. We used EMA with mobile phone applications to record the longitudinal contribution of these variables in predicting dietary temptation and lapse occurrences in a community sample of dieters in everyday life situations over a 7-day period. We examined both the direct and indirect (via temptation strength) relationships between these predictor variables and lapse occurrence. Another aim of this study was to examine how the aforementioned intrapersonal, situational, and psychological variables indirectly predict future self-efficacy via lapse occurrence. In other words, we examined how these variables predicted future self-efficacy to resist temptations by supporting or undermining current attempts to tackle temptations and prevent lapse. Having high levels of self-efficacy [17] has been repeatedly linked to weight-loss success [18-20]. However, Bandura argues that performance successes and failures are also linked to future self-efficacy. Investigating how 
dietary relapse is linked to future self-efficacy has been emphasized as a key area for weight loss research [10], nevertheless, the empirical evidence on this to date is scarce [1].

\section{Method}

\section{Participants}

The sample included 80 participants $\left(M_{\text {age }}=41.21 \pm 15.60\right.$ years; $M_{\text {Body Mass Index (BMI) }}=$ 30.78 $\pm 7.26,80 \%$ female). Weight status was established by inspecting current weekly recorded weights of the participants; in the absence of such records participants self-reported their most recent weight $(n=49)$. Participants were categorized by using their BMI as obese (33\%; BMI $\geq 30$ ), overweight (29\%; BMI $\geq 25)$, normal weight (17\%; BMI 18.5-24.9), severely/morbidly obese (14\%; BMI $\geq 35$ ), or their weight status was unspecified (7\%). Participants completed a screening questionnaire to ensure they were currently dieting to lose weight $(81.2 \%)$ or dieting to maintain their weight (18.8\%). The majority was in full-time employment (40\%); the remaining were university students (28\%), in part-time employment (14\%), retired (8\%), householders (5\%), unemployed (4\%), or their employment status was unspecified (1\%). Participants were White (80\%), South Asian (Indian, Pakistani and Bangladeshi; $12 \%$ ), Black (6\%), or of other ethnic origin (2\%). The majority of the participants’ highest qualification was high school education (30\%). Participants’ socioeconomic status (SES) was a continuous numerical score established based on current postcode and geo-demographic analysis software [21]. The mean SES score was 799 (SD = 143.16), just above the UK average of 750.

\section{Procedure}

Ethical approval for the study was granted by a British University ethics committee. Participants were recruited from local weight-loss groups (45\%) or were dieting on their own. Upon receipt of the screening questionnaire and informed consent, participants were invited 
to an individual meeting. During this meeting they completed a demographics questionnaire (i.e., height, weight-if not available via weight loss records-, age, gender, occupation, home postcode, and ethnicity). For descriptive purposes we administered measures of eating regulation intention [22], weight loss regulation for health versus appearance reasons [23], and degree of flexibility/rigidness of current weight loss practices [24]. We also distributed a number of questionnaires assessing dispositional factors which have been previously linked to weight maintenance success: trait self-control (Brief Self-Control Scale; BSCS;

Cronbach's $\alpha=.73$ [25], restrained, emotional and external eating behaviors (Dutch Eating Behaviors Questionnaire; DEBQ; Cronbach’s $\alpha=$.90) [26] perceived self-regulatory success in dieting (Perceived Self-Regulatory Success at Dieting scale; PSRS; Cronbach’s $\alpha=.65$ ) [16], and mindfulness (Mindful Attention Awareness Scale; Cronbach’s $\alpha=.89$ ) [27]. Given our focus on the daily predictors of dietary temptation and lapse, these variables were of secondary importance in this study and were treated as control variables.

Following questionnaire completion, participants were provided with a phone (Samsung B2100) which was enabled with a custom-made computer software application to record their daily dietary temptations for the next seven days. Each application contained a questionnaire with 15 items relating to the participants' recorded instances of dietary temptation and lapse. During the meeting all participants were given individual verbal and written instructions on how to recognize dietary temptations and lapses, how to record these on the phone application, and how to respond to the 15 questionnaire items. Dietary temptations were defined as “a sudden urge to overeat, eat a forbidden food, etc. in which you felt you had come close to the brink of breaking your diet”, and lapses were defined as “an incident where you felt that you broke your diet (e.g., overate, ate a forbidden food, etc.)', [6]. Participants were instructed to fill out the questionnaire on the phone as soon as possible or at least within 15 minutes of being tempted or having lapsed. After a week of 
diary entries participants returned the phone and completed a feedback questionnaire evaluating their compliance with the study procedures, using items adapted from Carels et al. [10].

We used an event-contingent as opposed to a signal-contingent (i.e., random prompts by the phone) methodology to record instances of dietary temptation and lapse. Random prompts by the device can result in missing episodes of temptation because of no prompts at these particular points in time. Furthermore, prompts by the device, especially when received in situations when no temptations are present, can increase participant burden and discontent about the study.

\section{Measures}

Phone diary. On experiencing a dietary temptation, participants were firstly required to indicate whether they lapsed by selecting Yes or No. Participants then responded to a series of 15 questions which tapped eight predictor variables (Table 1) based on previous weight loss and self-control research [6-12]. The questions examined the perceived strength of dietary temptation (e.g., “How strong was this dietary temptation?”), depletion (“How depleted did you feel prior to the dietary temptation?”), stress (“What was your level of stress immediately prior to the dietary temptation?”) and hunger (“How hungry were you immediately prior to the dietary temptation?”; all rated from 1, not very, to 7, extremely). Next, participants rated the role of situational factors on their dietary temptations (on a 1, not at all, to 7, very much, scale), such as the influence of others (e.g. "Did others influence your reaction to dietary temptation?”), and the environment in which the dietary temptation became available (whether the dietary temptation was unexpected (coded 0)-“This temptation became available to me unexpectedly”, or whether they sought for it (coded 1)-“I sought this temptation out”). Participants then rated their attempts to cope with the dietary temptation in terms of two 
coping strategies: Thinking long-term (“I thought about my long term weight loss goal”), and thinking about the importance of the goal ("I thought about how important weight loss is to me”; 1, not at all, to 7, very much). Finally, participants were asked, thinking of their current dietary temptation or lapse, to indicate their future self-efficacy ("How confident are you now in your ability resist future temptations?” ; 1= not very, to 7= extremely). With the exception of hunger, environment and coping, the other five variables (strength, stress, depletion, the presence of others, and future self-efficacy) were measured with two very similar items each and, therefore, their average score was used (average intra-variable $r$ across the 7 days $=$ $0.81)$.

\section{Data Analysis}

We employed multilevel modeling, using the MLwiN software (version 2.25) [28], as dietary temptation events were nested within individuals. Models were constructed to predict strength of dietary temptation, which was treated as a continuous outcome variable, and lapse occurrence which was treated as a binary ( $0=$ no lapse, $1=$ lapse) outcome variable. We firstly explored whether strength of dietary temptations or lapses were stronger/more frequent on weekdays or weekends, and in the morning, afternoon, or evening, by including these time period classifications as categorical independent variables in separate multilevel models $(5.00 \mathrm{am}-11.59 \mathrm{am}=1,12.00-4.59 \mathrm{pm}=2$, and 5.00pm-4.00am $)$.

Next, we examined whether a variety of demographic predictor variables were associated with strength of dietary temptation and lapse, respectively. Specifically, gender (coded as male $=0$, female $=1$ ), age, ethnicity (categorized as Asian, Black, White, and Other), socio-economic status, number of dependent children, and body mass index (BMI) were entered one at a time as predictors into the level 2 equation. Demographic variables were retained in the models if an association with the outcome variable was statistically significant. Building on these models, a number of dispositional variables (i.e. trait self- 
control, restrained eating, external eating, emotional eating, self-regulatory success in dieting, and mindfulness) were standardized, grand-mean centered, and entered as predictor variables, one at a time, into the level 2 equation. Only statistically significant associations among these dispositions and the two outcome variables were retained in the final multilevel models.

After establishing these baseline models, time-varying (level 1) scores of intrapersonal, situational, and psychological variables (Table 2) were included as predictors of strength of dietary temptation and lapse in the respective multilevel models. These variables were converted to z scores in order to obtain $\beta$ coefficients; they were also group-mean centered to provide pure estimates of the within-person associations and, importantly, to remove all between-person variance [29]. Based on these models, we also calculated the indirect relationships among the time-varying predictors and lapse occurrence, via the perceived strength of dietary temptation. As an indicator of effect size, $R_{1}{ }^{2}$ values were calculated for both dependent variables by comparing the final models with unconditional models. These values indicate the proportional amount of error of prediction reduced from the unconditional model to the final model at the within-person level [30]. To establish these values for the binary variable of lapse occurrence, an approximation of the level 1 variance term was established by treating lapse occurrence as a continuous variable [31].

Our next analysis tested whether the relationships among the statistically significant time-varying predictors of lapse occurrence (as identified in the analyses above), and the outcome variable of participants' self-efficacy to resist future dietary temptations were mediated by lapse occurrence. In line with the guidelines outlined by Krull and MacKinnon [32], we first examined whether the time-varying independent variables predicted the mediator (i.e., lapse occurrence). This was followed by identifying whether lapse occurrence predicted the outcome variable (i.e., future self-efficacy), after controlling for the 
independent variables. If these criteria were met, the significance of each indirect effect was examined using Sobel $Z$ scores.

\section{Results}

\section{Descriptive Analyses}

Participants reported 898 instances of dietary temptation, an average of 11.22 (SD $=.41$ ) dietary temptations per person over the seven day recording period. Of these instances, participants lapsed on average $52.4 \%$ of the time. Participants were more likely to give in to alcoholic temptations, compared to high sugar, and general excess consumption temptations ( $b=.69, p=.04$ and $b=.78, p=.04$, respectively). Average perceived strength of dietary temptation (measured from 1-7) was above moderate $(M=5.1 S D=1.37) ; 15 \%$ of dietary temptations were listed as 'extremely' strong. Table 1 presents the descriptive statistics of the daily intrapersonal, situational, and psychological factors included in this study.

On average participants had moderate to high intentions to regulate their eating ( $M=$ $5.28, S D=1.22$, on a 1-7 scale), and engaged in weight loss for health $(M=6.1, S D=0.9)$ and to a slightly lesser extent appearance motives $(M=5.0, S D=1.5$; both measured on a 1-7 scale). Finally participants tended to be flexible with their diets (rated from 1, strict and rigid diet, to 7, flexible and allowed for the occasional treat; $M=5.23, S D=1.54$ ). Table 2 presents the bivariate correlations among the study variables averaged over the course of the study. The two types of coping response were very highly correlated; therefore, they were combined into one composite coping response score in all analyses.

\section{Diary adherence and reactivity}

Upon returning their phone, participants were asked to complete a questionnaire measuring their adherence to the diary entries. On average, participants reported skipping 3.7 $(S D=2.80)$ no lapse entries and $1.30(S D=1.65)$ lapse entries throughout the seven day recording period. When asked how much keeping a diary influenced their eating behaviors, 
(30\%) indicated a little. The majority of participants either disagreed (46.7\%) or strongly disagreed (31. 1\%) with the statement that they were more tempted whilst keeping a diary; $52.3 \%$ and $29.5 \%$ either disagreed or strongly disagreed that they were more likely to lapse whilst keeping a diary. However, the majority of participants either strongly agreed (42\%) or agreed (28\%) that they were more aware of their behavior whilst keeping the diary.

\section{Do Strength of Dietary Temptation and Lapse Occurrence Vary as a Function of when the Dietary Temptation Occurred?}

No differences in the strength of dietary temptations were found at weekend compared to weekdays $(b=.02, p=.81)$, nor across mornings, afternoons, or evenings $(b=$ .24 to $.05, p=.06$ to .59$)$. Lapses were equally likely at weekend compared to weekdays $(b=$ $.02, p=.82)$, however, lapses were more likely in the evening compared to the morning $(b=$ $.37, p=.002)$ and afternoon $(b=.24, p=.01)$.

\section{Predictors of the Strength of Dietary Temptation and Lapse Occurrence}

None of the demographic or dispositional variables were significantly associated with perceived strength of dietary temptation or lapse; therefore, they were not included in subsequent analyses. We then included all the variables of primary interest in this study, namely the time-varying intra-personal, situational, and psychological predictors.

Standardized coefficients shown in Table 3 indicate that stress, hunger, the environment (whether the dietary temptation was unexpected), and other people being present, were positively associated with the strength of dietary temptation. Coping responses negatively predicted temptation strength and depletion did not predict the strength of dietary temptation. The final model explained $11 \%$ of the within-person variance in strength of dietary temptation. Regarding lapse occurrence, the strength of the dietary temptation, others being present, and the tempting environment increased the likelihood of lapsing. Moreover, coping 
strategies were negatively associated with lapse. Depletion, stress, and hunger were not associated with lapses. The final model explained $27 \%$ or the within-person variation in lapse occurrence. Calculation of indirect effects revealed that stress, hunger, the presence of others, coping responses and sought for temptation were indirectly associated with lapse occurrence, via the strength of the dietary temptation.

\section{Does Lapse Occurrence Mediate the Relationships among the Predictors of Lapse and Self-Efficacy to Resist Future Dietary Temptations?}

According to MacKinnon and Fairchild [33], direct relationships between the independent variable and the outcome are not necessary for testing mediation. Rather, in our first step in testing for this mediation, we ran a multilevel model that included only the significant predictors of lapse occurrence reported in Table 3. We did not include the nonsignificant predictors as the previous models established that they would not meet the first criterion in establishing mediation (i.e., the independent variable must significantly predict the mediator). In the second step we tested a model in which lapse occurrence significantly and negatively predicted self-efficacy to resist future dietary temptations, after controlling for the significant predictors in step $1(\beta=-.41, p<.001)$. This implied mediation effects, which were confirmed by statistically significant Sobel Z scores for indirect effects (Table 4). The strongest standardized indirect effects were found for coping response. We also tested the direct relations among the predictors and self-efficacy to resist future temptations. Results revealed that coping responses positively predicted self-efficacy $(\beta=.32, p<.001)$. In addition, seeking out the temptation negatively predicted future self-efficacy $(\beta=-.08, p=$ $.003)$.

\section{Supplementary Analyses}


We carried out a supplementary analysis examining whether future self-efficacy mediated the relationship between preceding and subsequent lapse occurrence later on the same day. For this analysis, we excluded days in which a participant experienced one dietary temptation only. Multilevel models using the steps described above showed no evidence for this mediation because self-efficacy did not predict future lapse occurrence after controlling for past lapse occurrence. We also repeated our analysis by excluding normal weight participants. The vast majority of results were the same as when all participants were included with the exception that whether or not one lapsed during the preceding temptation positively predicted subsequent lapse occurrence $(b=.36, p=.01$ versus $b=.15, p>.05$ in the whole sample).

\section{Discussion}

This study attempted to provide a socio-ecological perspective on dietary temptation and lapse by investigating the predictive role of various daily intrapersonal, psychological, and situational factors. This is the first study that examines how within-person changes in perceptions of a broad set of environmental and self-regulatory variables predict simultaneously within-person changes in dietary temptations and lapse occurrences using an EMA approach with mobile phones. Using multilevel modeling, we considered direct and indirect effects of these predictors in an initial attempt to build a coherent framework of dietary temptation and lapse.

Results indicated that dietary lapses occur frequently-just over $50 \%$ of the time our participants lapsed when tempted- a percentage which is approximately double than that reported by Hofmann et al. [8]. This difference might stem from discrepancies in the methodology (signal vs. event-contingent signaling) or the focus (the participants in Hofmann et al. monitored various temptations, whereas in the current investigation participants 
exclusively focused on dietary temptations) between the two studies. The majority of our participants reported that they were more aware of their behavior whilst keeping a diary, a finding also reported by Carels et al. [10]. The process of keeping a daily record of certain occurrences is a method of self-monitoring and self-monitoring has been repeatedly linked to increased behavioral awareness [34]. For example, asking about coping strategy options may prime participants to employ these strategies when normally they would not have used them and thus encourage them to overcome the urge to give in to dietary temptation [10]. Nevertheless, the average number of lapses recorded in the current study $(M=5.97, S D=$ .41) was considerably higher than that of other weight-related research $(M=2.7, S D=1.9$ [10], $M=1.5, S D=1.7$ [35]). The comparatively higher frequency seen in the current study lends support for the use of mobile phone applications for EMA investigations of dietary temptations and lapses.

\section{Daily Predictors of Perceived Dietary Temptation Strength and Lapse Occurrence}

We examined the predictive ability of various intrapersonal variables from the weight management and self-regulation literatures. We found that the stronger the dietary temptation, the more likely a participant was to lapse. This finding was similar to that of Hofmann et al. [8] who studied a number of different types of temptation. Levels of stress and hunger were found in our study to predict the strength of a temptation and indirectly lapses via temptation strength. Previous research has demonstrated a link between stress, hunger and food consumption $[6,12,15]$. Our findings are somewhat consistent with those reported in previous studies $[6,15]$ in that they show that the impact of stress and hunger on lapses is indirect via increasing the allure of the temptation.

The finding that depletion was not related to dietary temptation strength or lapse occurrence was inconsistent with that of previous research that linked depletion to diminished 
self-control and subsequent vulnerability to dietary temptation [11]. Previous studies examined depletion objectively by investigating differences between experimental and control groups in a self-control task (e.g. hand grip strength) following a depleting task (e.g. thought suppression or unsolvable anagrams) in which participants were blinded to the fact that their self-control was being depleted [11]. It is possible that the participants in our study were unable to recognize the signs of depletion and thus were unable to report it accurately. Indeed, our finding demonstrating differences in lapse occurrence over the course of a day provides support for this speculation. The finding that participants were most likely to lapse in the evening may indicate that they became depleted throughout the course of the day. This finding is also in line with Hoffman et al.' s [36] work.

Daily situational factors, namely the presence of others and the environment in which the participant was present (whether the dietary temptation was unexpected or whether participants sought for it) positively predicted the strength of dietary temptation and lapse occurrence. These findings are in agreement with previous research on relapse in the weight maintenance literature. Past research has shown that simply being around others increases susceptibility to dietary temptations and lapses $[8,15]$. It is thought that the presence of others influences not only what is eaten but how much is eaten [14]. Eating with familiar people is known to reduce an individual's ability and motivation to monitor consumption [14].

The current study also found that those temptations that were sought for had higher ratings of temptation strength and an increased likelihood of lapse, compared to dietary temptations that were unexpected. This is anticipated given that simply seeing tempting food is known to be associated with greater strength of a temptation [14]. This is thought to occur as the visibility of dietary temptations can act as a cue for consumption. Thus, by exposing oneself to dietary temptation the likelihood of lapsing increases [14]. Lapse occurrence and strength of temptation were negatively associated with the use of the specific coping 
mechanisms of thinking of one's long-term weight loss goal and considering the importance of their goal. These findings are consistent with past research which found that dietary lapses were associated with lower levels of coping efforts $[6,15]$. This is the first study to examine the aforementioned coping mechanisms using EMA.

Lapse occurrence was also found to negatively predict self-efficacy to resist future dietary temptations. This finding is expected given that self-efficacy is not only the confidence in one's ability to overcome obstacles and accomplish goals, but also entails expectations of future success based on past performance accomplishments or failures [20]. There is a substantial amount of research linking self-efficacy and weight loss success [1820]. However, the current study is among the first to demonstrate the link between momentary lapse occurrence and future self-efficacy. Indeed, improving an individual's responses following lapse (i.e. whether they see it as a small mistake vs. feeling terrible and going off the diet) "may be the single-most-effective way of preparing people to maintain their weight loss” [37]. Our mediation analysis further shows that underscoring the importance of seeing lapses as temporary and reinforcing positive coping responses (such as those detailed above), can positively predict perceptions of future self-efficacy following lapse. In contrast, the presence of others in tempting situations and unexpected dietary temptations undermine one’s self-efficacy to resist future dietary temptations.

No significant relationships were found between temptation strength or lapse occurrence and any of the dispositional factors examined in the study. Our findings are somewhat similar to those of Hofmann et al. [8], who found that certain dispositional variables (e.g. trait self-control, perfectionism, narcissistic tendencies) were related to factors that occurred in the middle of their 4-step model (conflict and resistance), but unrelated to the final stage, that is behavioral enactment. For example, individuals with high trait self-control make attempts to control their environment to avoid dietary temptations and conflict with 
other goals, and this might explain why trait self-control was unrelated to lapse occurrence in the present study.

\section{Implications}

The effects found in the current study could potentially help piece together the complex jigsaw surrounding the daily predictors of dietary temptations and help us to better understand how dietary temptations and lapses operate. As such these findings have a number of implications for future practice. For example, they allude to the potential utility of future specialized mobile phone applications that can be used as monitoring tools for individuals on weight management programs so that they can record personal and situational circumstances under which they experience dietary temptations and/or lapses. In relation to situational factors, the findings of the current study indicate the potential for dietary relapse programs to highlight the danger of social influence and norms in terms of a dieter's vulnerability to dietary temptation. The results also demonstrate the importance of the adoption of specific coping mechanisms to help prevent lapses, and highlight the significance of targeting an individual's self-efficacy following lapse. Improvement of both self-efficacy and coping responses could have a substantial impact on weight loss success. One might ask whether lapses are always perceived as negative, or whether some degree of dietary "lapse" is expected in the course of dieting. Although one should try and reduce lapses, realistically these cannot be eliminated completely. Indeed our participants indicated that they were not very rigid with their dieting, and such flexibility might be important for long-term success.

\section{Limitations and Future Research Directions}

Our study was subject to a few limitations. First, a small proportion of our sample consisted of participants who were of 'normal weight' (17\%); although these may have been previously overweight dieters striving to maintain their weight, the experience of dietary 
temptations in this group may differ from those who are overweight or obese. However, we re-ran the analyses without normal weight participants and the results were largely the same with the noticeable exception that whether or not overweight and obese participants lapsed during the preceding temptation positively predicted subsequent lapse occurrence on the same day. Additionally, rather than simply measuring if others were present Hofmann et al. [8] found that the presence of others engaging in the desired behavior was more influential on behavioral enactment (giving in to temptation) than others being present but not engaging in the desired behavior. Future work should include this important distinction and also examine the weight status of others to see if this is influential on lapse occurrence. A further limitation of our work was that the event-contingent recording did not allow for the assessment of events that are not connected to temptation. Hence, it was not possible to determine the relative differences between tempting and non-tempting situations and ascertain what is unique about the former situations. Future research could combine event- and signalcontingent recordings to address this question. A further limitation of the event-contingent methodology (which we chose so that participants would not miss out on any temptation episodes) is that it could have actually resulted in more temptations and eventually lapses being reported than usual, because participants were constantly thinking about having to monitor their behavior. An additional limitation of our work was that we measured whether participants lapsed but not the severity of lapse (i.e., amount of food consumed). It would be interesting for further research to examine what degree of lapse is considered expected and/or appropriate by participants in the course of dieting and how this is linked to long-term weight loss success or failure.

Future research could also utilize longer time frameworks and examine whether weight loss success and failure predict differential rates of self-efficacy, temptation, and lapse. More importantly, future research should continue our efforts to integrate the various 
predictors of dietary temptations and lapse into a coherent socio-ecological framework. Such attempts require additional evidence using a variety of methodologies and contexts.

\section{Conclusion}

In the fight against obesity we need to help individuals become more aware of the various personal, situational, and environmental factors that expose them to dietary temptations, or instead help them to develop the necessary skills to cope successfully with dietary temptations and prevent lapses. The current study adds in different ways to what is already known in the literature and its findings could be utilized in future dietary relapse and weight maintenance programs.

Conflict of interest statement: The authors have no conflict of interest to disclose. 


\section{References}

1. Green AR, Larkin M, Sullivan V. “Oh stuff it!” The experience and explanation of diet failure: An exploration using interpretative phenomenological analysis. Journal of Health Psychology. 2009;14:997-1008. doi:10.1177/1359105309342293.

2. Byrne S, Cooper Z, Fairburn C. Weight maintenance and relapse in obesity: a qualitative study. International Journal of Obesity. 2003; 27:955-62. doi:10.1038/sj.ijo.0802305.

3. Crescioni W, Ehrlinger J, Alquist JL, et al. High trait self-control predicts positive health behaviors and success in weight loss. Journal of Health Psychology. 2011;16:750-9. doi:10.1177/1359105310390247.

4. Duckworth AL. The significance of self-control. Proceedings of the National Academy of Sciences of the United States of America. 2011;108:2639-40. doi:10.1073/pnas.1019725108.

5. Harvey EL, Summerbell CD, Kirk SFL, Hill AJ. Dietitians’ views of overweight and obese people and reported management practices. Journal of Human Nutrition and Dietetics. 2002;15:331-47.

6. Carels R, Hoffman J, Collins A, Raber A, Cacciapaglia H, O’Brien W. Ecological momentary assessment of temptation and lapse in dieting. Eating Behaviors. 2001;2:307321. doi:10.1016/S1471-0153(01)00037-X.

7. Muraven M, Baumeister RF. Self-regulation and depletion of limited resources: Does selfcontrol resemble a muscle? Psychological Bulletin. 2000;126:247-259. doi:10.1037//00332909.126.2.247. 
8. Hofmann W, Baumeister RF, Förster G, Vohs KD. Everyday temptations: An experience sampling study of desire, conflict, and self-control. Journal of Personality and Social Psychology. 2012;102:1-18. doi:10.1037/a0026545.

9. Stone AA, Shiffman S. Capturing momentary, self-report data: A proposal for reporting guidelines. Annals of Behavioral Medicine. 2002;24:236-243.

doi:10.1207/S15324796ABM2403_09.

10. Carels R, Douglass O, Cacciapaglia HM, O’Brien WH. An ecological momentary assessment of relapse crises in dieting. Journal of Consulting and Clinical Psychology. 2004;72:341-8. doi:10.1037/0022-006X.72.2.341.

11. Baumeister RF, Bratslavsky E, Muraven M, Tice DM. Ego depletion: Is the active self a limited resource? Journal of Personality and Social Psychology. 1998;74:1252-1265. doi:10.1037//0022-3514.74.5.1252.

12. Royal JD, Kurtz JL. I ate what?! The effect of stress and dispositional eating style on food intake and behavioral awareness. Personality and Individual Differences. 2010;49:565-569. doi:10.1016/j.paid.2010.04.022.

13. Hagger MS, Wood C, Stiff C, Chatzisarantis NLD. Ego depletion and the strength model of self-control: a meta-analysis. Psychological Bulletin. 2010;136:495-525. doi:10.1037/a0019486.

14. Wansink B. Environmental factors that increase the food intake and consumption volume of unknowing consumers. Annual Review of Nutrition. 2004;24:455-79. doi:10.1146/annurev.nutr.24.012003.132140. 
15. Grilo CM, Shiffman S, Wing RR. Relapse crises and coping among dieters. Journal of Consulting and Clinical Psychology. 1989;57:488-495. doi:10.1037//0022-006X.57.4.488.

16. Fishbach A, Friedman RS, Kruglanski AW. Leading us not into temptation: Momentary allurements elicit overriding goal activation. Journal of Personality and Social Psychology. 2003;84:296-309. doi:10.1037/0022-3514.84.2.296.

17. Bandura A. Self-efficacy: The exercise of control. New York: Freeman; 1997.

18. Kitsantas A. The role of self-regulation strategies and self-efficacy perceptions in successful weight loss maintenance. Psychology \& Health. 2000;15:811-820. doi:10.1080/08870440008405583.

19. Byrne S. Psychological aspects of weight maintenance and relapse in obesity. Journal of Psychosomatic Research. 2002;53:1029-1036. doi:10.1016/S0022-3999(02)00487-7.

20. Elfhag K, Rössner S. Who succeeds in maintaining weight loss? A conceptual review of factors associated with weight loss maintenance and weight regain. Obesity Reviews. 2005;6:67-85. doi:10.1111/j.1467-789X.2005.00170.x.

21. Cameo. 2012. Available at: http://www.checkmyfile.com/postcode-check/SW1A2AA.htm .

22. Pelletier LG, Dion SC, Slovinec-D’Angelo M, \& Reid R. Why Do You Regulate What You Eat? Relationships Between Forms of Regulation, Eating Behaviors, Sustained Dietary Behavior Change, and Psychological Adjustment. Motivation and Emotion. 2004; 28: 245277. doi:10.1023/B:MOEM.0000040154.40922.14 
23.Verstuyf J, Vansteenkiste M, Soenens, B. Eating regulation and bulimic symptoms: The differential correlates of health-focused and appearance-focused eating regulation. Body Image. 2011;9: 108-117. doi:10.1016/j.bodyim.2011.09.003

24.Westenhoefer J, Stunkard A J, \& Pudel V. Validation of the flexible and rigid control dimensions of dietary restraint. International Journal of Eating Disorders, 1999; 26:53-64. DOI:10.1002/(SICI)1098-108X(199907)26:1<53::AID-EAT7>3.0.CO;2-N

25. Tangney JP, Baumeister RF, Boone AL. High self-control predicts good adjustment, less pathology, better grades, and interpersonal success. Journal of personality. 2004;72:271-324. 26. Van Strien T, Frijters JER, Bergers GP., Defares PB. The Dutch Eating Behavior Questionnaire (DEBQ) for assessment of restrained, emotional, and external eating behavior. International Journal of Eating Disorders. 1986;5:295-315.

27. Tapper K, Shaw C, Ilsley J, Hill AJ, Bond, FW, \& Moore L.. Exploratory randomized controlled trial of a mindfulness-based weight loss intervention for women. Appetite. 2009; 52: 396-404. doi:10.1016/j.appet.2008.11.012

28. Rasbash J, Browne WJ, Healy M, Cameron B, Charlton CMJ. MLwiN Version 2.25. 2012.

29. Enders CK, Tofighi D. Centering predictor variables in cross-sectional multilevel models: a new look at an old issue. Psychological Methods. 2007;12:121-38. doi:10.1037/1082989X.12.2.121.

30. Hox J. Multilevel analysis. Techniques and applications. 2nd Editio. New York: Routledge; 2010. 
31. Goldstein H, Rasbash J, Browne W. Partitioning variation in multilevel models. Understanding Statistics. 2002;1:223-231.

32.Krull JL, MacKinnon DP. Multilevel Modeling of Individual and Group Level Mediated Effects. Multivariate Behavioral Research. 2001;36:249-277. doi:10.1207/S15327906MBR3602_06.

33. Fairchild AJ, MacKinnon DP. A general model for testing mediation and moderation effects. Prevention science : the official journal of the Society for Prevention Research. 2009;10:87-99. doi:10.1007/s11121-008-0109-6.

34.Burke LE, Wang J, Sevick MA. Self-monitoring in weight loss: a systematic review of the literature. Journal of the American Dietetic Association. 2011;111:92-102. doi:10.1016/j.jada.2010.10.008.

35.Carels RA, Cacciapaglia HM, Rydin S, Douglass OM, Harper J. Can social desirability interfere with success in a behavioral weight loss program? Psychology \& Health. 2006;21:65-78. doi:10.1080/14768320500102277.

36. Hofmann W, Vohs KD, \& Baumeister, RF. What people desire, feel conflicted about, and try to resist in everyday life. Psychological Science. 2012; 23: 582-8. doi:10.1177/0956797612437426

37. Dohm FA, Beattie JA, Aibel C, Striegel-Moore RH. Factors differentiating women and men who successfully maintain weight loss from women and men who do not. Journal of Clinical Psychology. 2001;57:105-17. doi:10.1002/1097-4679(200101)57:1<105::AIDJCLP11>3.0.CO;2-I. 
Table 1

Descriptive Statistics of Daily Predictors of No Lapse and Lapse

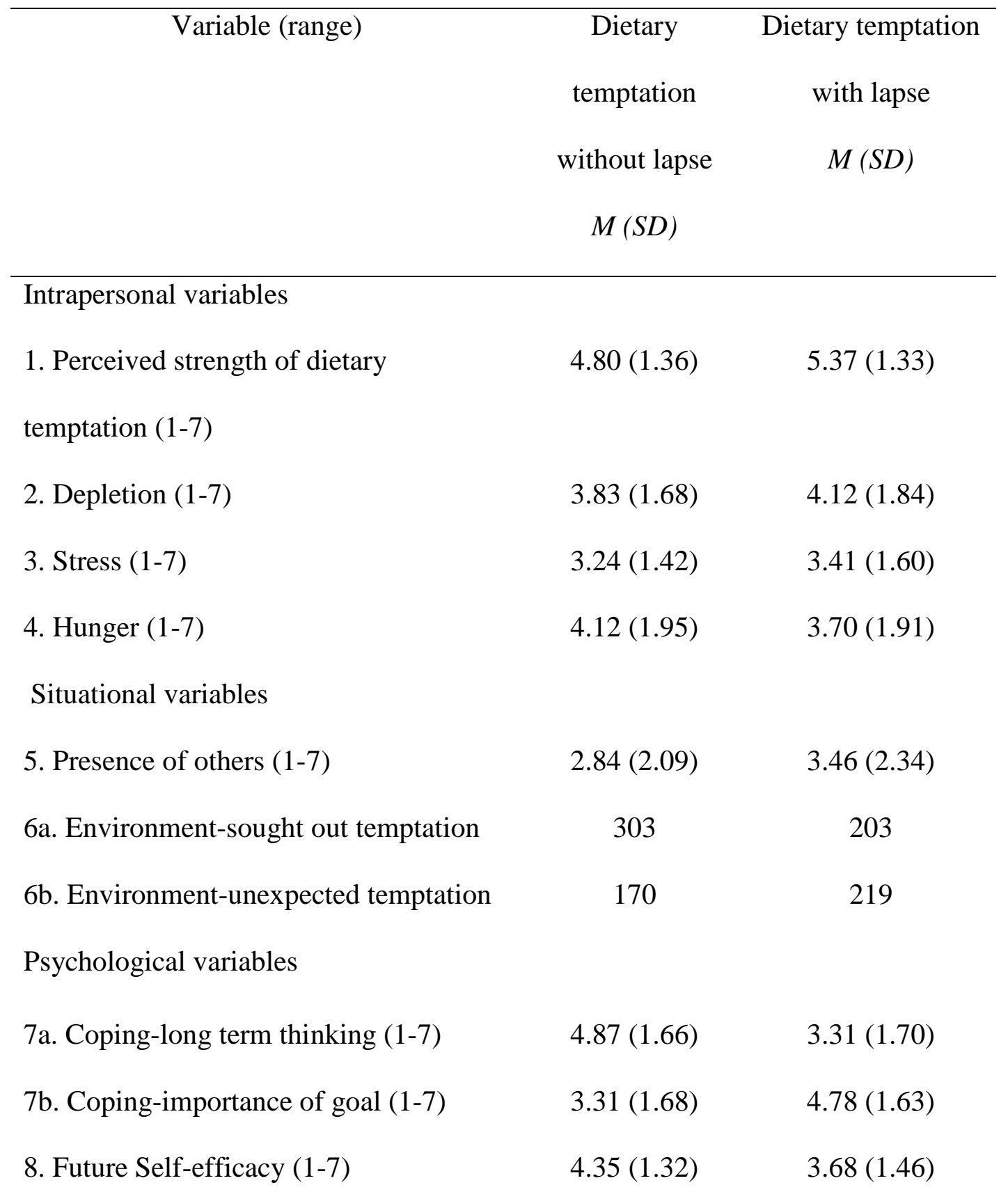

Note. ${ }^{1}$ Environment was a binary variable; participants had to choose one of two options: $0=$ temptation unexpected, 1 = temptation sought out. Thus, for this variable frequencies rather than means and standard deviations are presented. 
Table 2

Bivariate Correlations among Daily Predictors across the Course of the Study

\begin{tabular}{|c|c|c|c|c|c|c|c|c|c|}
\hline Variable & 1. & 2. & 3. & 4. & 5. & 6. & 7. & 8. & 9. \\
\hline 1. Strength of temptation & - & & & & & & & & \\
\hline 2. Depletion & $.11^{* *}$ & - & & & & & & & \\
\hline 3. Stress & $.11^{* *}$ & $.59 * *$ & - & & & & & & \\
\hline 4. Hunger & $.22 * *$ & $.17^{* *}$ & $.16^{* *}$ & - & & & & & \\
\hline 5. Presence of others & $.17 * *$ & .05 & .05 & .03 & - & & & & \\
\hline 6. Environment & $.12^{* *}$ & $.11^{* *}$ & .03 & $.08 *$ & $-.17 * *$ & - & & & \\
\hline 7. Coping: Long-term thinking & $-.08 *$ & $-.08 *$ & -.01 & $.11^{* *}$ & $-.09 * *$ & -.05 & - & & \\
\hline 8. Coping: Importance of goal & -.06 & -.04 & .00 & $-.09 * *$ & $-.09 * *$ & -.04 & $.90 * *$ & - & \\
\hline 9. Future self-efficacy & .02 & $.11^{* *}$ & -.04 & -.02 & .04 & $-.08^{*}$ & $.44^{* *}$ & $.48 * *$ & - \\
\hline 10. Coping Composite & $-.07 *$ & -.06 & -.01 & $-.10 * *$ & $-.09 * *$ & -.05 & $.98 * *$ & $.97 * *$ & \\
\hline
\end{tabular}

Note. ${ }^{*} p<.05$, ** $p<.01$. Environment refers to whether temptation was sought out or unexpected. 
Table 3

Final Multilevel Models Exploring Predictors of Strength of Dietary temptation and Lapse Occurrence

Outcome Variable

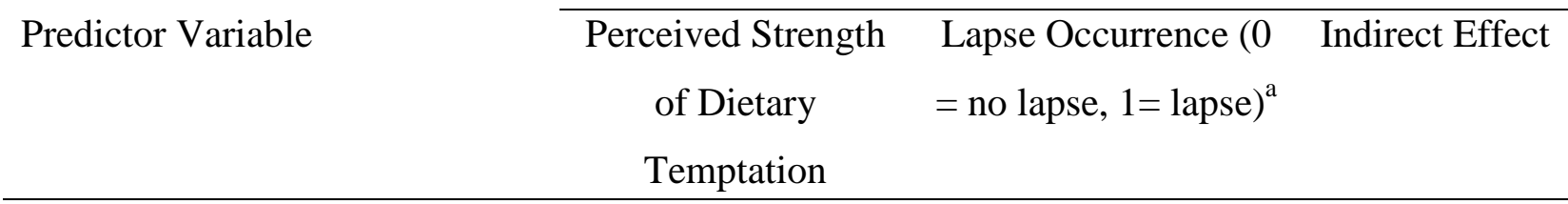

Fixed Effects

Intercept

$5.263^{* * *}$

$0.151^{*}$

Intrapersonal Variables

Perceived strength of $.198 * *$

temptation

Depletion

$-.023$

.087

$-.004$

Stress

$.176^{* *}$

.017

$.034 *$

Hunger

$.278^{* * *}$

.048

$.055^{* *}$

Situational Variables

Presence of others

$.129 * *$

$.172^{* *}$

$.025 *$

Environment (unexpected vs.

$.137^{* *}$

$.162^{* *}$

$.027 *$

sought out temptation)

Coping responses

$-.254 * * *$

$-.763 * * *$

$-.050 * *$

Random Effects
Intercept
$.428 * * *$
Level 1 error
$1.331^{* * *}$
$\mathrm{R}_{1}^{2}$
.109
$.204^{* *}$
.268
Note. ${ }^{*} p<.05,{ }^{* *} p<.01,{ }^{* * *} p<.001{ }^{\text {a }}$ Regression weights for predicting lapse occurrence are log-odds. The Level 1 error term is not reported for the logistic model because no single variance value can be obtained in binary response models ${ }^{26}$ 
Table 4

Multilevel Models Exploring Whether the Significant Intrapersonal, Situational, and Psychological Variables in Table 3 Indirectly Predict Self-Efficacy to Resist Future Dietary temptations via Lapse Occurrence

\section{Mediation Step $1 \quad$ Mediation Step 2}

Predictor Variable

Outcome: Lapse

Outcome: Self- Indirect Effects

Occurrence $(0=$ no

Efficacy

lapse, $1=$ lapse) ${ }^{\mathrm{a}}$

\section{Effects}

Intercept

Perceived strength of

temptation

Presence of others

Environment (unexpected vs.

sought out temptation)

Coping responses

Lapse occurrence

Random Effects

Intercept

Level 1 error

$\mathrm{R}_{1}^{2}$
$0.152 *$

$221 * * *$

$.170^{* *}$

$.170 * * *$

$-.762 * * *$

$.204^{* *}$

$-$

.264
$3.965 * * *$

$-.038$

$-.091 * * *$

.008

$-.070 * *$

$-.056^{*}$

$-.070 * *$

$.212 * * *$

$.314 * * *$

$-.412 * * *$

$1.563 * * *$

$.410 * * *$

.212

Note. ${ }^{*} p<.05,{ }^{* *} p<.01,{ }^{* * *} p<.001{ }^{\mathrm{a}}{ }^{\mathrm{a}}$ Regression weights for predicting lapse occurrence are log-odds. 